\title{
Comparing the efficacy of induction-concurrent with concurrent-adjuvant chemotherapy in locoregionally advanced nasopharyngeal carcinoma: a propensity score matching analysis
}

\author{
Li-Rong Wu $\mathbf{u}^{1, *}$, Xue-Song Jiang ${ }^{1, *}$ Xue Song ${ }^{1}$, Hong-Liang Yu ${ }^{1}$, Yan-Xin Fan ${ }^{1}$, Fei- \\ Jiang Wang ${ }^{1}$, Sheng-Fu Huang ${ }^{1}$, Wen-Jie Guo ${ }^{1}$, Xia $\mathrm{He}^{1}$ and Ju-Ying Liu ${ }^{1}$ \\ ${ }^{1}$ Department of Radiation Oncology, Nanjing Medical University Affiliated Cancer Hospital, Jiangsu Cancer Hospital and \\ Jiangsu Institute of Cancer Research, Nanjing 210009, China \\ *These authors contributed equally to this work \\ Correspondence to: Xia He, email: hexia206@yeah.net \\ Ju-Ying Liu, email: juyingliu@ $21 \mathrm{cn} . c 0 m$
}

Keywords: nasopharyngeal carcinoma, locoregionally advanced, induction chemotherapy, adjuvant chemotherapy, intensity-modulated radiotherapy

Received: June 21, $2017 \quad$ Accepted: August 06, $2017 \quad$ Published: August 22, 2017

Copyright: Wu et al. This is an open-access article distributed under the terms of the Creative Commons Attribution License 3.0 (CC BY

3.0), which permits unrestricted use, distribution, and reproduction in any medium, provided the original author and source are credited.

\section{ABSTRACT}

Purpose: This study aimed to compare the efficacy of induction-concurrent (IC-CCRT) with concurrent-adjuvant (CCRT-AC) chemotherapy in patients with locoregionally advanced nasopharyngeal carcinoma (LA-NPC) treated by intensitymodulated radiotherapy (IMRT).

Materials and Methods: Data on 834 patients with newly diagnosed, nonmetastatic stage III-IVA (except T3NO) NPC receiving either IC-CCRT or CCRT-AC between July, 2004 and December, 2014 were retrospectively reviewed. Propensity score matching (PSM) method was adopted to balance prognostic factors and match patients. Survival outcomes of matched patients between IC-CCRT and CCRT-AC were compared.

Results: The median follow-up duration is 45.2 months (range, 1.07-145.4 months). Overall, 309 pairs were selected by PSM. Univariate analysis revealed the CCRT-AC group achieved significantly higher 3-year DFS (83.9\% vs. $78.7 \%$; $P=$ $0.014)$ and $O S(87.6 \%$ vs. $87.0 \% ; P=0.031)$. Multivariate analysis also identified treatment group (IC-CCRT vs. CCRT-AC) as an independent prognostic factor for 3-year DFS (HR, 1.546; 95\% CI, 1.113-2.149; $P=0.009)$ and OS (HR, 1.487; 95\% CI, 1.035-2.136; $P=0.032$ ). Subgroup analysis revealed IC-CCRT was a protective factor for DMFS (HR, 0.145; 95\% CI, 0.043-0.488; P = 0.002) in stage III disease; however, it could adversely affected DFS (HR, 2.009; 95\% CI, 1.316-3.065; $P=$ $0.001)$, OS (HR, 1.671; 95\% CI, 1.060-2.636; $P=0.027)$ and DMFS (HR, $1.986 ; 95 \%$ CI, 1.155-3.416; $P=0.013$ ) in stage IVA disease.

Conclusions: CCRT-AC may be a more effective treatment modality in patients with stage IVA NPC disease, while IC-CCRT was superior in stage III disease.

\section{INTRODUCTION}

As an Epstein-Barr virus (EBV)-associated malignant tumor [1-3], nasopharyngeal carcinoma (NPC) has an extremely unbalanced distribution: 86700 new cases were reported with $71 \%$ of those occurring in east and southeast parts of Asia in 2012 [4]. Apart from other head and neck cancers, radiotherapy is the only curative treatment modality for non-metastatic NPC due to its radiosensitivity and anatomical constraint which makes radical surgery unavailable. Also, NPC is highly sensitive to chemotherapy, and combined chemotherapyradiotherapy strategies have been documented to achieve better survival outcomes than radiotherapy alone in 
advanced NPC [5-12]. Consequently, concurrent chemoradiotherapy (CCRT) has become the basic treatment for locoregionally advanced NPC (LA-NPC). However, CCRT may be insufficient as survival of LANPC is mainly compromised by distant metastasis, especially for patients receiving intensity-modulated radiotherapy (IMRT) $[13,14]$. Therefore, additional chemotherapy to CCRT is urgently needed.

Since the Intergroup-0099 trial [5] showed a benefit of 3-year overall survival provided by CCRT plus adjuvant chemotherapy (AC), this regimen has been recommended as the standard care for advanced NPC. However, most patients suffering severe toxicities during CCRT and could not tolerate the toxicities of $\mathrm{AC}$, which constrains its wide use. Therefore, a strategy with better efficacy and less toxicities should be developed to improve compliance to treatment and systemic control. Induction chemotherapy (IC), delivered before CCRT, has attracted a lot of attention as it has better compliance and could eliminate micro-metastasis. For the last twenty years, much efforts have been made regarding the value of IC [15-23]; however, most of these studies achieved negative results except for the studies by Hui et al. [19] and by Sun et al. [23]. Besides, a pooled data analysis by Chua et al. [24] revealed IC was associated with significantly decreased disease-specific survival, which indicating that IC may still play an important role in LA-NPC.

Given the abovementioned evidence, there has come a concern: which chemotherapy sequence is better? Induction with concurrent (IC-CCRT) or concurrent followed by adjuvant (CCRT-AC)? However, little is known about the head-to-he comparison of these two regimens except the results from two network metaanalysis $[25,26]$. Accordingly, we conducted this retrospective study to compare the survival difference between these treatment modalities.

\section{RESULTS}

\section{Baseline characteristics}

From July 2004 to December 2014, we identified 834 patients with LA-NPC (except T3N0) receiving either IC-CCRT or CCRT-AC, among whom 487 (58.4\%) received IC-CCRT and $347(41.6 \%)$ received CCRTAC. For the whole cohort, the male $(n=641)$-to-female $(n=193)$ ratio was $3.3: 1$, and the median age was 48 (18-70) years-old. After matching by PSM, 309 pairs were selected from the original 834 patients and the baseline characteristics were summarized in Table 1. Obviously, patients in these two groups received similar treatment intensity (chemotherapy regimen and cycle). Moreover, host and tumor stage factors were also well balanced between these two groups after matching $(P>0.05$ for all rates).

\section{Treatment failure patterns}

Up to the last visit (July 2016), the median followup duration was 45.2 months (range, 1.07-145.4 months) for the selected cohort. Notably, 73 (23.6\%) patients in the IC-CCRT group and $49(15.9 \%)$ in the CCRT-AC group $\operatorname{died}(P=0.015)$. Among the 122 deaths, there were 16 $(13.1 \%)$ non-cancer deaths with $12(9.8 \%)$ and $4(3.3 \%)$ in IC-CCRT and CCRT-AC groups, respectively. Moreover, $48(15.5 \%)$ patients in IC-CCRT group and $39(12.6 \%)$ in the CCRT-AC group developed distant metastasis $(P=0.298)$. Particularly, $30(9.7 \%)$ in IC-CCRT group and $20(6.5 \%)$ in CCRT-AC group experienced locoregional recurrence $(P=0.140)$. Overall, $87(28.2 \%)$ patients in the IC-CCRT and $60(19.4 \%)$ in CCRT-AC group suffered treatment failure $(P=0.011)$.

\section{Univariate and multivariate analysis}

Univariate analysis revealed the estimated 3-year DFS, OS, DMFS and LRRFS rates were $81.3 \%, 87.3 \%$, $87.9 \%$ and $93.7 \%$ for the whole cohort. Compared with the CCRT-AC group, the IC-CCRT group achieved significantly worse 3 -year DFS $(78.7 \%$ vs. $83.9 \%$, $P=0.014$; Figure $1 \mathrm{~A})$ and $\mathrm{OS}(87.0 \%$ vs. $87.6 \%$, $P=0.031$; Figure 1B); however, 3-year DMFS $(86.7 \%$ vs. $89.1 \%, P=0.270$; Figure $1 \mathrm{C})$ and LRRFS (92.7\% vs. $94.7 \%, P=0.128$; Figure 1D) were comparable between these two groups.

After adjusting for various factors, multivariate analysis revealed treatment group (IC-CCRT vs. CCRT$\mathrm{AC}$ ) was an independent prognostic factor for 3-year DFS (HR, 1.546; 95\% CI, 1.113-2.149; $P=0.009)$ and OS (HR, 1.487 ; 95\% CI, 1.035-2.136; $P=0.032)$. However, it did not have a survival impact on 3-year DMFS (HR, 1.294; 95\% CI, 0.847-1.976; $P=0.233$ ) and LRRFS (HR, 1.530; 95\% CI, 0.869-2.696; $P=0.141$ ) (Table 2). Undoubtedly, overall stage (IVA vs. III) was an independent prognostic factor for all endpoints.

\section{Subgroup analysis}

To further establish the survival difference of these treatment modalities in patients at different risk, we therefore conducted stratified analysis according to the tumor stage because it was established as an independent prognostic factor by multivariate analysis.

In primary cohort, 407 patients had stage III disease and 147 pairs were selected for this analysis. The 3-year DFS, OS, DMFS and LRRFS rates for the IC-CCRT vs. CCRT-AC group were $88.7 \%$ vs. $85.4 \%(P=0.107$; Figure $2 \mathrm{~A}), 93.7 \%$ vs. $89.7 \%(P=0.261$; Figure $2 \mathrm{~B}), 97.9 \%$ vs. $88.3 \%$ $(P<0.001 ;$ Figure $2 \mathrm{C})$ and $91.7 \%$ vs. $97.0 \%$ $(P=0.376$; Figure 2D), respectively. When multivariate 
Table 1: Baseline characteristics of the 309 pairs with LA-NPC (except T3N0)

\begin{tabular}{|c|c|c|c|}
\hline Characteristics & $\begin{array}{c}\text { IC + CCRT } \\
\text { No. }(\%)\end{array}$ & $\begin{array}{c}\text { CCRT + AC } \\
\text { No. }(\%)\end{array}$ & $P$ \\
\hline Median age (y, range) & $47(18-70)$ & $48(18-69)$ & $0.213^{\mathrm{a}}$ \\
\hline Gender & & & $0.296^{\mathrm{b}}$ \\
\hline Male & $258(83.5)$ & $248(80.3)$ & \\
\hline Female & $51(16.5)$ & $61(19.7)$ & \\
\hline KPS & & & $0.468^{\mathrm{b}}$ \\
\hline$\geq 90$ & $222(71.8)$ & $230(74.4)$ & \\
\hline$\leq 80$ & $87(28.2)$ & $79(25.6)$ & \\
\hline Smoking & & & $0.934^{\mathrm{b}}$ \\
\hline Yes & $116(37.5)$ & $117(37.9)$ & \\
\hline No & $193(62.5)$ & $192(62.1)$ & \\
\hline Drinking & & & $0.378^{\mathrm{b}}$ \\
\hline Yes & $53(17.2)$ & $45(14.6)$ & \\
\hline No & $256(82.8)$ & $264(85.4)$ & \\
\hline T category ${ }^{\mathrm{c}}$ & & & $0.348^{b}$ \\
\hline $\mathrm{T} 1$ & $19(6.1)$ & $30(9.7)$ & \\
\hline $\mathrm{T} 2$ & $25(8.1)$ & $25(8.1)$ & \\
\hline $\mathrm{T} 3$ & $130(42.1)$ & $116(37.5)$ & \\
\hline $\mathrm{T} 4$ & $135(43.7)$ & $138(44.7)$ & \\
\hline $\mathrm{N}$ category ${ }^{\mathrm{c}}$ & & & $0.470^{\mathrm{b}}$ \\
\hline No & $18(5.8)$ & $15(4.9)$ & \\
\hline N1 & $164(53.1)$ & $153(49.5)$ & \\
\hline $\mathrm{N} 2$ & $89(28.8)$ & $107(34.6)$ & \\
\hline N3 & $38(12.3)$ & $34(11.0)$ & \\
\hline Overall stage $\mathrm{e}^{\mathrm{c}}$ & & & $0.835^{\mathrm{b}}$ \\
\hline III & $142(46.0)$ & $146(47.2)$ & \\
\hline IVA & $167(54.0)$ & $163(52.8)$ & \\
\hline CCRT regimen & & & $0.489^{\mathrm{b}}$ \\
\hline $\mathrm{PF}$ & $94(30.4)$ & $102(33.0)$ & \\
\hline $\mathrm{TP}$ & $215(69.6)$ & $207(67.0)$ & \\
\hline IC/AC cycles & & & $0.747^{\mathrm{b}}$ \\
\hline 2 & $170(55.0)$ & $166(53.7)$ & \\
\hline 3 & $139(45.0)$ & $143(46.3)$ & \\
\hline $\mathrm{IC} / \mathrm{AC}$ regimen & & & $0.723^{\mathrm{b}}$ \\
\hline $\mathrm{TPF}$ & $88(28.5)$ & $94(30.4)$ & \\
\hline $\mathrm{TP}$ & $156(50.5)$ & $146(47.2)$ & \\
\hline PF & $65(21.0)$ & $69(23.4)$ & \\
\hline
\end{tabular}

Abbreviations: LA-NPC = locoregionally advanced nasopharyngeal carcinoma; IC $=$ induction chemotherapy; CCRT = concurrent chemoradiotherapy; $\mathrm{AC}=$ adjuvant chemotherapy; KPS = karnofsky performance score; IMRT = intensitymodulated radiotherapy.

${ }^{a} P$-value was calculated by Non-parametric test.

${ }^{\mathrm{b}} P$-values were calculated by Chi-square test.

${ }^{\mathrm{c}}$ According to the 8 th AJCC/UICC staging system. 
analysis was performed, IC-CCRT group was found to be superior to CCRT-AC group with regard to DMFS (HR, 0.145; 95\% CI, 0.043-0.488; $P=0.002$ ), while the other endpoints were comparable between the two groups (Table 3).

Among the 427 patients with stage IVA disease, 157 pairs were selected. The 3-year DFS, OS, DMFS, LRRFS rates in the IC-CCRT and CCRT-AC groups were $73.0 \%$ and $81.0 \%(P=0.001$; Figure $3 \mathrm{~A}), 85.6 \%$ and $85.5 \%(P=0.019$; Figure $3 \mathrm{~B}), 79.9 \%$ and $88.4 \%$ $(P=0.011$; Figure $3 C), 94.3 \%$ and $92.2 \%(P=0.304$; Figure 3D), respectively. Obviously, patients in the CCRT-AC group achieved significantly better DFS, OS and DMFS. And consistent with the results of univariate analysis, treatment group (IC-CCRT vs. CCRT-AC) was established as a survival predictive factor for DFS (HR, 2.009; 95\% CI, 1.316-3.065; $P=0.001$ ), OS (HR, 1.671; 95\% CI, 1.060-2.636; $P=0.027)$ and DMFS (HR, 1.986; $95 \% \mathrm{CI}, 1.155-3.416 ; P=0.013)$ but not for LRRFS (HR, $1.445 ; 95 \%$ CI, 0.693-3.013; $P=0.326$ ) (Table 3 ).

\section{DISCUSSION}

Notably, our study to date is the first head-to-head study with the largest cohorts to compare the therapeutic
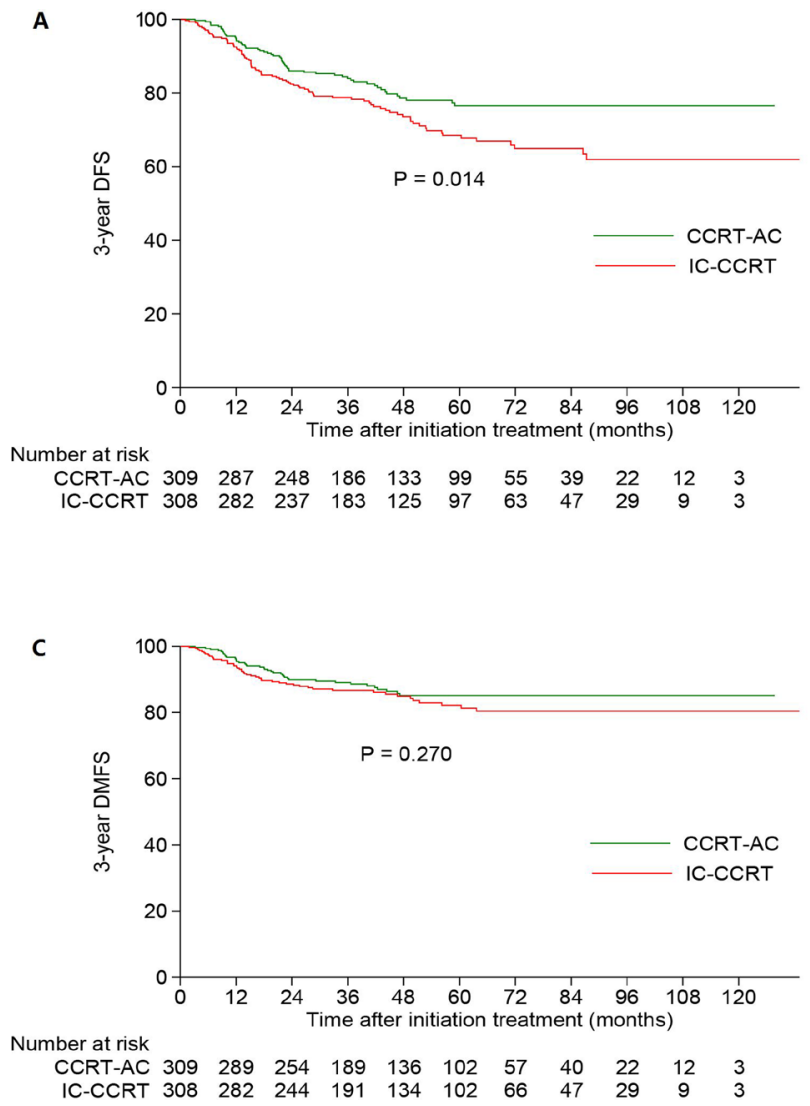

gain achieved by IC-CCRT or CCRT-AC in LA-NPC (except T3N0). The results demonstrated that CCRT-AC chemotherapy sequence was associated with significantly improved DFS and OS compared with IC-CCRT, and this benefit may mainly originate from the cohort of patients with stage IVA disease. With the advantages of employing PSM and multivariate analysis, this study provided the fairest comparisons of matched patients and the outcomes were robust.

CCRT followed by adjuvant fluorouracil with cisplatin has been recommended as the standard treatment strategy for LA-NPC by National Comprehensive Cancer Network (NCCN) guidelines since the publication of Intergroup-0099 trial [5], and many subsequent studies carried out in Asia further established the value of this chemotherapy regimen $[9,10,12,27]$. However, this regimen brought severe toxicities and the compliance to three cycles in previously reported studies was unsatisfactory $(52-63 \%)$ [5, 9, 10, 12, 27, 28]. Therefore, a strategy with better efficacy and less toxicities is needed to improve compliance to treatment and systemic control. Consequently, much attention has been paid to induction chemotherapy as it has satisfactory compliance and could shrink tumor volume and eliminate micro-metastasis before radiotherapy. Many trials achieved positive results

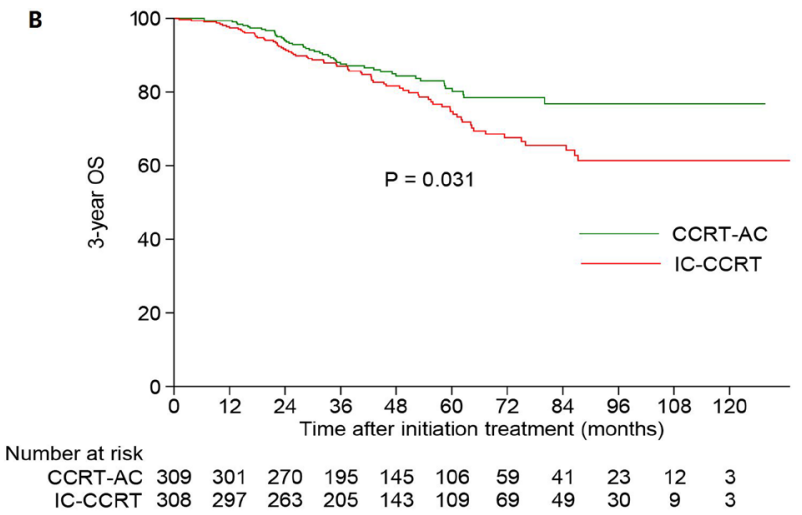

D

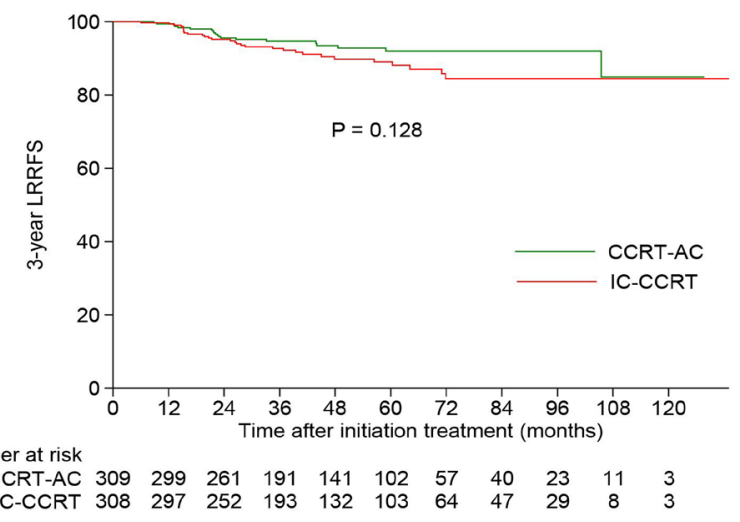

Figure 1: Kaplan-Meier DFS (A) OS (B), DMFS (C), and LRRFS (D) curves for 309 pairs of patients stratified as IC-CCRT and CCRT-AC groups. Abbreviations: OS = overall survival; DFS = disease-free survival; DMFS = distant metastasis-free survival; LRRFS = locoregional relapse-free survival; $\mathrm{IC}=$ induction chemotherapy; CCRT = concurrent chemoradiotherapy; $\mathrm{AC}=$ adjuvant chemotherapy. 
Table 2: Results of multivariate analysis for the 309 pairs with LA-NPC (except T3N0)

\begin{tabular}{llcc}
\hline Endpoints & \multicolumn{1}{c}{ Variables } & HR (95\% CI) & $\boldsymbol{P}^{\mathbf{a}}$ \\
\hline DFS & Smoking (Yes vs. no) & $1.867(1.345-2.591)$ & $<0.001$ \\
& Treatment group (IC-CCRT vs. CCRT-AC) & $1.546(1.113-2.149)$ & 0.009 \\
& Overall stage (IVA vs. III) & $2.287(1.610-3.247)$ & $<0.001$ \\
OS & Smoking (Yes vs. no) & $2.254(1.574-3.228)$ & $<0.001$ \\
& Treatment group (IC-CCRT vs. CCRT-AC) & $1.487(1.035-2.136)$ & 0.032 \\
\multirow{2}{*}{ DMFS } & Overall stage (IVA vs. III) & $2.421(1.641-3.572)$ & $<0.001$ \\
& Smoking (Yes vs. no) & $1.578(1.028-2.422)$ & 0.037 \\
& Treatment group (IC-CCRT vs. CCRT-AC) & $1.294(0.847-1.976)$ & 0.233 \\
& T category (T3-4 vs. T1-2) & $2.266(1.094-4.693)$ & 0.028 \\
& N category (N2-3 vs. N0-1) & $2.096(1.345-3.266)$ & 0.001 \\
LRRFS & Overall stage (IVA vs. III) & $2.254(1.415-3.591)$ & 0.001 \\
& Treatment group (IC-CCRT vs. CCRT-AC) & $1.530(0.869-2.696)$ & 0.141 \\
\hline
\end{tabular}

Abbreviations: LA-NPC $=$ locoregionally advanced nasopharyngeal carcinoma; DFS $=$ disease-free survival; OS $=$ overall survival; DMFS = distant metastasis-free survival; LRRFS = locoregional relapse-free survival; HR $=$ hazards ratio; $\mathrm{CI}=$ confidence interval; KPS $=$ Karnofsky performance score; IC $=$ induction chemotherapy; CCRT $=$ concurrent chemoradiotherapy; $\mathrm{AC}=$ adjuvant chemotherapy.

${ }^{a}$ Multivariate $P$-values were calculated using an adjusted Cox proportional-hazards model with backward elimination and the following parameters: age ( $>48$ y vs. $\leq 48$ y), gender (female vs. male), KPS ( $\geq 90$ vs. $\leq 80$ ), smoking (yes vs. no), drinking (yes vs. no), T category (T3-4 vs. T1-2), $\mathrm{N}$ category (N2-3 vs. N0-1), overall stage (IVA vs. III), concurrent chemotherapy regimen (TP vs. PF), treatment group (IC-CCRT vs. CCRT-AC).
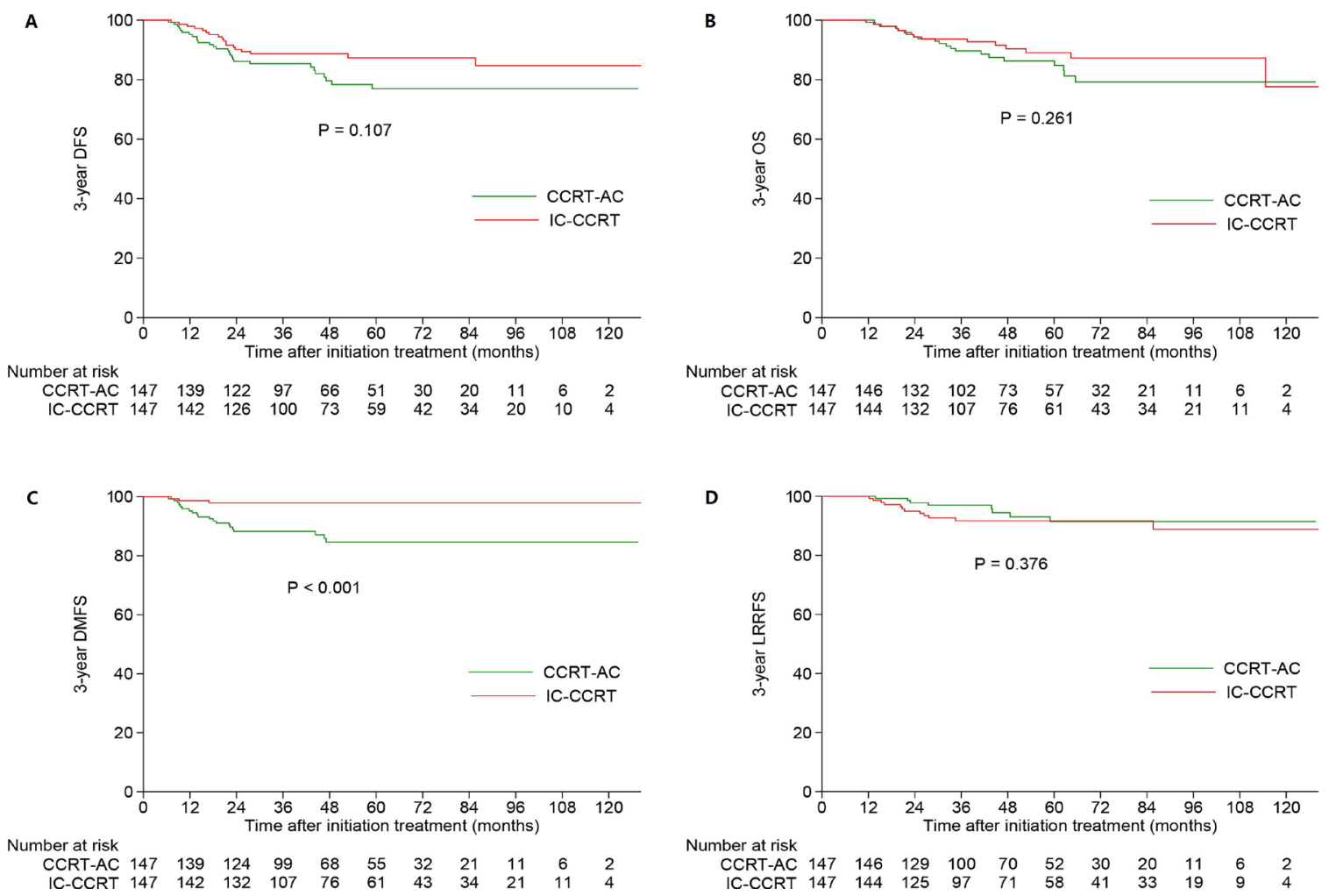

Figure 2: Kaplan-Meier DFS (A), OS (B), DMFS (C) and LRRFS (D) curves for patients with stage III receiving IC-CCRT or CCRTAC. Abbreviations: OS = overall survival; DFS = disease-free survival; DMFS = distant metastasis-free survival; LRRFS = locoregional relapse-free survival; IC = induction chemotherapy; CCRT = concurrent chemoradiotherapy; AC = adjuvant chemotherapy. 
Table 3: Results of multivariate analysis in different subgroups

\begin{tabular}{llcc}
\hline \multicolumn{1}{c}{ Endpoints } & \multicolumn{1}{c}{ Variable } & HR (95\% CI) & $\boldsymbol{P}^{\mathbf{a}}$ \\
\hline Stage III cohort & & & \\
DFS & N category (N2 vs. N1) & $2.030(1.080-3.815)$ & 0.028 \\
& Treatment group (IC-CCRT vs. CCRT-AC) & $0.617(0.341-1.116)$ & 0.111 \\
OS & KPS ( $\geq$ 90 vs. $\leq$ 80) & $0.298(0.104-0.854)$ & 0.024 \\
& N category (N2 vs. N1) & $1.887(1.045-3.407)$ & 0.035 \\
& Treatment group (IC-CCRT vs. CCRT-AC) & $0.704(0.361-1.373)$ & 0.303 \\
DMFS & N category, N2 vs. N1 & $2.409(1.199-4.839)$ & 0.014 \\
\multirow{2}{*}{ LRRFS } & Treatment group (IC-CCRT vs. CCRT-AC) & $0.145(0.043-0.488)$ & 0.002 \\
Stage IVA cohort & Treatment group (IC-CCRT vs. CCRT-AC) & $1.431(0.578-3.539)$ & 0.438 \\
DFS & & & \\
& Smoking (Yes vs. No) & $3.239(1.820-5.763)$ & $<0.001$ \\
& Treatment group (IC-CCRT vs. CCRT-AC) & $2.009(1.316-3.065)$ & 0.001 \\
OS & N category (N2-3 vs. N0-1) & $1.622(1.083-2.428)$ & 0.019 \\
& Smoking (Yes vs. No) & $0.254(0.09-0.714)$ & $<0.001$ \\
& Treatment group (IC-CCRT vs. CCRT-AC) & $1.671(1.060-2.636)$ & 0.027 \\
DMFS & N category (N2-3 vs. N0-1) & $1.648(1.062-2.560)$ & 0.026 \\
& Smoking (Yes vs. No) & $2.210(1.314-3.718)$ & 0.003 \\
LRRFS & Treatment group (IC-CCRT vs. CCRT-AC) & $1.986(1.155-3.416)$ & 0.013 \\
\hline A & N category (N2-3 vs. N0-1) & $2.066(1.209-3.530)$ & 0.008 \\
\hline
\end{tabular}

Abbreviations: DFS = disease-free survival; OS = overall survival; DMFS = distant metastasis-free survival; LRRFS = locoregional relapse-free survival; $\mathrm{HR}=$ hazards ratio; $\mathrm{CI}=$ confidence interval; IC $=$ induction chemotherapy; CCRT = concurrent chemoradiotherapy; $\mathrm{AC}=$ adjuvant chemotherapy; KPS = karnofsky performance score.

${ }^{a}$ Multivariate $P$-values were calculated using an adjusted Cox proportional-hazards model with backward elimination and the following parameters: age ( $>48$ y vs. $\leq 48$ y), gender (female vs. male), KPS ( $\geq 90$ vs. $\leq 80$ ), smoking (yes vs. no), drinking (yes vs. no), T category (T3-4 vs. T1-2), N category (N2-3 vs. N0-1), concurrent chemotherapy regimen (TP vs. PF) and treatment group (IC-CCRT vs. CCRT-AC).

and made induction chemotherapy a more promising treatment modality $[15,19,23,29]$. However, little is known about the comparison of these two regimens since no randomized trial has been undertaken except the study consisting of six arms by Lee et al. [20] and two network meta-analysis $[25,26]$. In the current study, we presented that CCRT-AC may be a more effective option than IC-CCRT in decreasing death and treatment failure for patients with stage III-IVA (except T3N0) disease. The underlying reason for this difference may be that patients in the IC-CCRT group experience longer wait time before definitive radiotherapy which could do harm to survival $[25,30]$.

Stratified analysis according to the tumor stage (III or IVA) was performed as it was an independent prognostic factor indicated by multivariate analysis. Intriguingly, absolutely different outcomes were observed in these two subpopulations. In the cohort of stage III, a better DMFS rate was obtained in the IC-CCRT group although the other endpoints were non-significant. However, CCRTAC could achieved better DFS and OS compared with
IC-CCRT in patients with stage IV disease. Two potential factors may contribute to the different results. First, tumor burden and risk of distant metastasis is higher in the stage IVA disease than that in stage III disease. The negative effects of prolonged wait time on survival outcomes may be amplified and more obvious in these patients with higher risk of metastasis. Moreover, compared with the $\mathrm{PF}$ regimen used in previous studies [5, 9, 12, 28, 31], the chemotherapy regimen used in our study in the CCRTAC cohort were TPF and TP which were more effective than the PF regimen. Therefore, it may be likely that TPF or TP works in the adjuvant phase better than in the induction phase. Notably, the DFS rate at the 3-year point was higher in the CCRT-AC group than that in IC-CCRT group but OS rate ( $85.6 \%$ vs. $85.5 \%)$ was similar in the stage IVA subpopulation. The reason may be attributed to the effective salvage treatment for patients experienced treatment failure after IC-CCRT. Although OS rate at 3 -year point was comparable, a significant difference $(P=0.019)$ was still obtained and that is because more patients experienced treatment failure after 3 years. 
Therefore, these results may indicate that CCRT-AC treatment still has a strong protective effect after 3 years.

It should be pointed out that the concurrent chemotherapy regimen used in our study is double agents consisting of TP and PF, which originated from the study by $\mathrm{Lin}$ et al. [11]. Although, double-agent regimens may had more toxicities than single agent of cisplatin, we only assigned two cycles and most of patients could completed that. Moreover, multivariate analysis reveal the chemotherapy regimen (TP vs. PF) used in concurrent phase was not a prognostic factor for all endpoints. Therefore, the concurrent chemotherapy should have no or very limited impact on the conclusions.

The main strength of this study is the adoption of PSM and multivariate analysis to evaluate the survival difference between patient receiving IC-CCRT and CCRTAC chemotherapy sequences in LA-NPC; this addressed the potential limitations of divergent confounders, treatment heterogeneity and selection bias associated with retrospective analysis [32]. As with all retrospective studies, the weakness of this study should also be acknowledged. First, the data was collected from a single institution and toxicity data was unavailable because
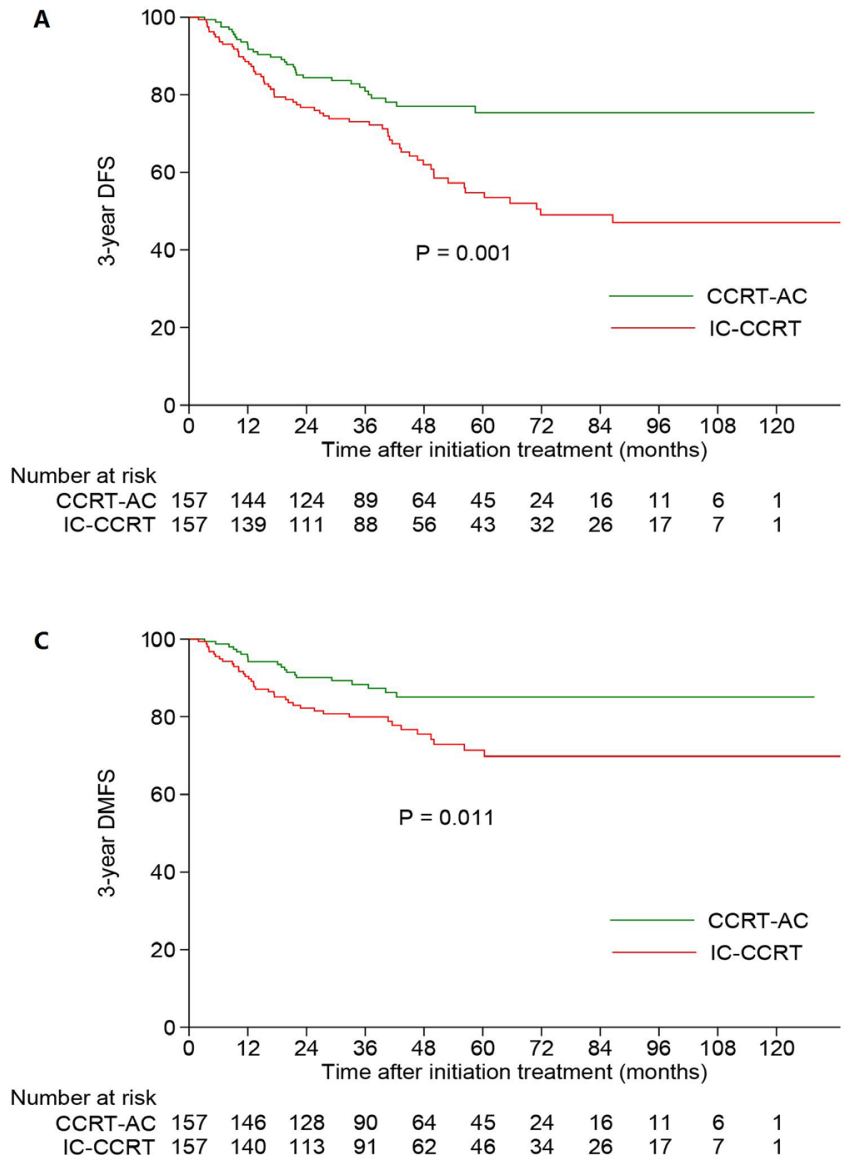

our retrospective study did not collected this. Moreover, prognostic biomarker such as plasma Epstein-Barr virus (EBV) DNA [3, 33-35] was not given consideration because part of the data were collected at a very early time when detection of plasma EBV DNA was unavailable. Future clinical trials regarding plasma EBV DNA should be conducted for better risk stratification when comparing these two treatment modalities.

\section{MATERIALS AND METHODS}

\section{Patient selection}

We retrospectively reviewed data on patients with newly diagnosed, non-metastatic NPC receiving IMRT between July, 2004 and December, 2014 at Nanjing Medical University Affiliated Cancer Hospital of China. Patients meeting the following criteria were recruited for this study: (1) stage III-IVA NPC (except T3N0); (2) World Health Organization (WHO) pathology type II/III; (3) receiving either IC-CCRT or CCRT-AC; (3) age 18-70 years older; (4) without previous malignancy; (5) did not receiving chemotherapy or/and radiotherapy previously.
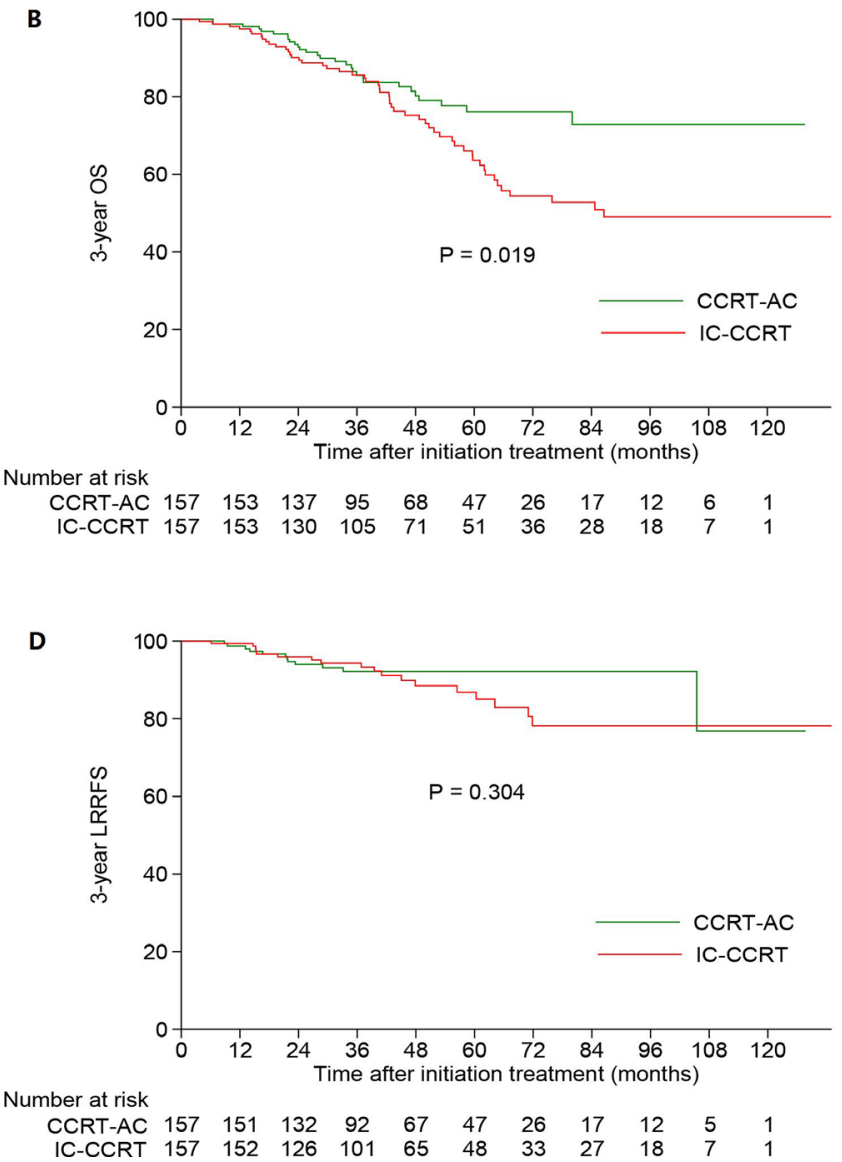

Figure 3: Kaplan-Meier DFS (A), OS (B), DMFS (C) and LRRFS (D) curves for patients with stage IVA receiving IC-CCRT or CCRTAC. Abbreviations: OS = overall survival; DFS = disease-free survival; DMFS = distant metastasis-free survival; LRRFS = locoregional relapse-free survival; IC = induction chemotherapy; CCRT = concurrent chemoradiotherapy; AC = adjuvant chemotherapy. 
Finally, 834 patients were identified. This study was approved by the Research Ethics Committee of Jiangsu Cancer Hospital. Written informed consent was obtained from all the patients before treatment.

\section{Staging workup}

The routine staging workup before treatment contained a complete history collecting, clinical examinations of the head and neck region and direct fibreoptic nasopharyngoscopy. Radiographic examinations included magnetic resonance imaging (MRI) or contrastenhanced computed tomography (CT) scans of the skull base and whole neck, chest radiography, whole-body bone scan and abdominal sonography, as well as positron emission tomography (PET)-CT if necessary. All MRI materials and clinical records were reviewed to minimize heterogeneity in restaging, and all patients were restaged according to the 8th edition of the International Union against Cancer/American Joint Committee on Cancer (UICC/AJCC) system [36].

\section{Radiotherapy}

Definitive IMRT was delivered to all the patients with $6 \mathrm{MV}$ X-rays in our center as reported previously [37]. Briefly, gross tumor volume (GTVnx) included the primary tumor and metastatic retropharyngeal lymph nodes. Metastatic cervical lymph nodes were defined as GTVnd. The high-risk region was defined as clinical target volume (CTV1) which included the whole nasopharyngeal cavity, GTVnx, GTVnd with a margin of 5 to $15 \mathrm{~mm}$, and levels II and III cervical lymphatic drainage region. Low risk area was defined as CTV2 which encompassed CTV1 with a margin of 3 to $5 \mathrm{~mm}$, the lower neck, and the supraclavicular lymphatic drainage region. A total prescribed dose of 66-75 Gy/31-35 fractions to the planning target volume (PTV) of GTVnx, 65-75 Gy/3235 fractions to the PTV of GTVnd, 56-60Gy/30 fractions to the PTV of CTV1 and 50 Gy/30 fractions to the PTV of CTV2, respectively. All patients were irradiated with 1 fraction daily, 5 days per week.

\section{Chemotherapy}

IC or AC were platinum-based chemotherapy regimens including 5-fluorouracil $\left(1000 \mathrm{mg} / \mathrm{m}^{2} \mathrm{~d} 1\right.$ d5) with cisplatin ( $80 \mathrm{mg} / \mathrm{m}^{2}$ in total for 3 days) (PF), docetaxel $\left(75 \mathrm{mg} / \mathrm{m}^{2} \mathrm{~d} 1\right)$ with cisplatin $\left(80 \mathrm{mg} / \mathrm{m}^{2}\right.$ in total for 3 days) (TP) or triplet of docetaxel $\left(60 \mathrm{mg} / \mathrm{m}^{2}\right.$ d1) plus cisplatin $\left(80 \mathrm{mg} / \mathrm{m}^{2}\right.$ in total for 3 days) with 5-fluorouracil (1000 mg/m² d1-d5) (TPF), which were administered every three weeks for 2-3 cycles. Concurrent chemotherapy consisted of two cycles of TP or PF and the dosage was delivered as abovementioned.

\section{Follow-up}

Follow-up duration was measured from first day of pathological diagnosis to last examination or death. Patients were assessed every 3 months during the first 2 years, then every 6 months thereafter (or until death) by clinical examinations, abdominal sonography, MRI of nasopharynx and neck and chest X-ray or CT. PET-CT was also performed if clinical symptoms indicated distant metastasis. End points analysed in our study included 3-year disease-free survival (DFS), overall survival (OS), distant metastasis-free survival (DMFS) and locoregional relapse-free survival (LRRFS).

\section{Statistical analysis}

Propensity score matching (PSM) [32] was computed by logistic regression for each patient using the following covariates: age, gender, karnofsky performance score (KPS), smoking, drinking, T category, N category, overall stage, IC/AC regimen and cycle, and CCRT regimen. Chi-square test or Fisher's exact test were employed to compare categorical variables and treatment failure patterns between IC-CCRT and CCRT-AC groups. Non-parametric test was used to compare continuous variables. Survival rates were estimated using the KaplanMeier method and the difference was compared by logrank test. The multivariate Cox proportional hazards model was performed to estimate hazard ratios (HRs) and $95 \%$ confidence intervals (CIs); age ( $>44$ y vs. $\leq 44$ y), gender (female vs. male), KPS ( $\geq 90$ vs. $\leq 80$ ), smoking (yes vs. no), drinking (yes vs. no), T category (T3-4 vs. T1-2), N category (N2-3 vs. N0-1), overall stage (IVA vs. III), concurrent chemotherapy regimens (TP vs. PF), treatment group (IC-CCRT vs. CCRT-AC). All tests were two-sided; $P<0.05$ was considered significant. Stata Statistical Package 12 (StataCorp LP, College Station, TX, USA) was used for all analyses.

\section{Grant support}

This work was funded by the National Natural Science Foundation of China (No. 81672989), Jiangsu Clinical medicine Science and Technology Special Fund (BL2014091), and Jiangsu Provincial Commission of Health and Family Planning Youth Research Project (Q201601).

\section{CONCLUSIONS}

Overall, our findings suggest that CCRT-AC treatment modality could achieve better therapeutic outcomes compared with IC-CCRT in the era of IMRT and this benefit may mainly originate from the patients with stage IVA, while IC-CCRT may be considered for stage 
III disease. Future randomized trials consisting more risk stratification factors are warranted to confirm our results.

\section{CONFLICTS OF INTEREST}

The authors declare no competing interest.

\section{REFERENCES}

1. Chan AS, To KF, Lo KW, Mak KF, Pak W, Chiu B, Tse GM, Ding M, Li X, Lee JC, Huang DP. High frequency of chromosome $3 p$ deletion in histologically normal nasopharyngeal epithelia from southern Chinese. Cancer Res. 2000; 60:5365-5370.

2. Pathmanathan R, Prasad U, Sadler R, Flynn K, RaabTraub N. Clonal proliferations of cells infected with EpsteinBarr virus in preinvasive lesions related to nasopharyngeal carcinoma. N Eng J Med. 1995; 333:693-698.

3. Lin JC, Wang WY, Chen KY, Wei YH, Liang WM, Jan JS, Jiang RS. Quantification of plasma Epstein-Barr virus DNA in patients with advanced nasopharyngeal carcinoma. N Eng J Med. 2004; 350:2461-2470.

4. Torre LA, Bray F, Siegel RL, Ferlay J, Lortet-Tieulent J, Jemal A. Global cancer statistics, 2012. CA Cancer J Clin. 2015; 65:87-108.

5. Al-Sarraf M, LeBlanc M, Giri PG, Fu KK, Cooper J, Vuong T, Forastiere AA, Adams G, Sakr WA, Schuller DE, Ensley JF. Chemoradiotherapy versus radiotherapy in patients with advanced nasopharyngeal cancer: phase III randomized Intergroup study 0099. J Clin Oncol. 1998; 16:1310-1317.

6. Chan AT, Leung SF, Ngan RK, Teo PM, Lau WH, Kwan WH, Hui EP, Yiu HY, Yeo W, Cheung FY, Yu KH, Chiu KW, Chan DT, et al. Overall survival after concurrent cisplatin-radiotherapy compared with radiotherapy alone in locoregionally advanced nasopharyngeal carcinoma. J Natl Cancer Inst. 2005; 97:536-539.

7. Chan AT, Teo PM, Ngan RK, Leung TW, Lau WH, Zee B, Leung SF, Cheung FY, Yeo W, Yiu HH, Yu KH, Chiu KW, Chan DT, et al. Concurrent chemotherapy-radiotherapy compared with radiotherapy alone in locoregionally advanced nasopharyngeal carcinoma: progression-free survival analysis of a phase III randomized trial. J Clin Oncol. 2002; 20:2038-2044.

8. Kwong DL, Sham JS, Au GK, Chua DT, Kwong PW, Cheng AC, Wu PM, Law MW, Kwok CC, Yau CC, Wan KY, Chan RT, Choy DD. Concurrent and adjuvant chemotherapy for nasopharyngeal carcinoma: a factorial study. J Clin Oncol. 2004; 22:2643-2653.

9. Lee AW, Lau WH, Tung SY, Chua DT, Chappell R, Xu L, Siu L, Sze WM, Leung TW, Sham JS, Ngan RK, Law SC, Yau TK, et al. Preliminary results of a randomized study on therapeutic gain by concurrent chemotherapy for regionallyadvanced nasopharyngeal carcinoma: NPC-9901 Trial by the Hong Kong Nasopharyngeal Cancer Study Group. J Clin Oncol. 2005; 23:6966-6975.

10. Lee AW, Tung SY, Chua DT, Ngan RK, Chappell R, Tung R, Siu L, Ng WT, Sze WK, Au GK, Law SC, O’Sullivan B, Yau TK, et al. Randomized trial of radiotherapy plus concurrent-adjuvant chemotherapy vs radiotherapy alone for regionally advanced nasopharyngeal carcinoma. J Natl Cancer Inst. 2010; 102:1188-1198.

11. Lin JC, Jan JS, Hsu CY, Liang WM, Jiang RS, Wang WY. Phase III study of concurrent chemoradiotherapy versus radiotherapy alone for advanced nasopharyngeal carcinoma: positive effect on overall and progression-free survival. J Clin Oncol. 2003; 21:631-637.

12. Wee J, Tan EH, Tai BC, Wong HB, Leong SS, Tan T, Chua ET, Yang E, Lee KM, Fong KW, Tan HS, Lee KS, Loong S, et al. Randomized trial of radiotherapy versus concurrent chemoradiotherapy followed by adjuvant chemotherapy in patients with American Joint Committee on Cancer/International Union against cancer stage III and IV nasopharyngeal cancer of the endemic variety. J Clin Oncol. 2005; 23:6730-6738.

13. Lai SZ, Li WF, Chen L, Luo W, Chen YY, Liu LZ, Sun Y, Lin AH, Liu MZ, Ma J. How does intensitymodulated radiotherapy versus conventional twodimensional radiotherapy influence the treatment results in nasopharyngeal carcinoma patients? Int J Radiat Oncol Biol Phys. 2011; 80:661-668.

14. Sun X, Su S, Chen C, Han F, Zhao C, Xiao W, Deng X, Huang S, Lin C, Lu T. Long-term outcomes of intensity-modulated radiotherapy for 868 patients with nasopharyngeal carcinoma: an analysis of survival and treatment toxicities. Radiother Oncol. 2014; 110:398-403.

15. Chua DT, Sham JS, Choy D, Lorvidhaya V, Sumitsawan Y, Thongprasert S, Vootiprux V, Cheirsilpa A, Azhar T, Reksodiputro AH. Preliminary report of the Asian-Oceanian Clinical Oncology Association randomized trial comparing cisplatin and epirubicin followed by radiotherapy versus radiotherapy alone in the treatment of patients with locoregionally advanced nasopharyngeal carcinoma. AsianOceanian Clinical Oncology Association Nasopharynx Cancer Study Group. Cancer. 1998; 83:2270-2283.

16. Fountzilas G, Ciuleanu E, Bobos M, Kalogera-Fountzila A, Eleftheraki AG, Karayannopoulou G, Zaramboukas T, Nikolaou A, Markou K, Resiga L, Dionysopoulos D, Samantas E, Athanassiou H, et al. Induction chemotherapy followed by concomitant radiotherapy and weekly cisplatin versus the same concomitant chemoradiotherapy in patients with nasopharyngeal carcinoma: a randomized phase II study conducted by the Hellenic Cooperative Oncology Group (HeCOG) with biomarker evaluation. Ann Oncol. 2012; 23:427-435.

17. Hareyama M, Sakata K, Shirato H, Nishioka T, Nishio M, Suzuki K, Saitoh A, Oouchi A, Fukuda S, Himi T. A prospective, randomized trial comparing neoadjuvant chemotherapy with radiotherapy alone in patients with 
advanced nasopharyngeal carcinoma. Cancer. 2002; 94:2217-2223.

18. Hong RL, Ting LL, Ko JY, Hsu MM, Sheen TS, Lou PJ, Wang CC, Chung NN, Lui LT. Induction chemotherapy with mitomycin, epirubicin, cisplatin, fluorouracil, and leucovorin followed by radiotherapy in the treatment of locoregionally advanced nasopharyngeal carcinoma. J Clin Oncol. 2001; 19:4305-4313.

19. Hui EP, Ma BB, Leung SF, King AD, Mo F, Kam MK, Yu BK, Chiu SK, Kwan WH, Ho R, Chan I, Ahuja AT, Zee BC, et al. Randomized phase II trial of concurrent cisplatin-radiotherapy with or without neoadjuvant docetaxel and cisplatin in advanced nasopharyngeal carcinoma. J Clin Oncol. 2009; 27:242-249.

20. Lee AW, Ngan RK, Tung SY, Cheng A, Kwong DL, Lu TX, Chan AT, Chan LL, Yiu H, Ng WT, Wong F, Yuen KT, Yau S, et al. Preliminary results of trial NPC-0501 evaluating the therapeutic gain by changing from concurrent-adjuvant to induction-concurrent chemoradiotherapy, changing from fluorouracil to capecitabine, and changing from conventional to accelerated radiotherapy fractionation in patients with locoregionally advanced nasopharyngeal carcinoma. Cancer. 2015; 121:1328-1338.

21. Ma J, Mai HQ, Hong MH, Min HQ, Mao ZD, Cui NJ, Lu TX, Mo HY. Results of a prospective randomized trial comparing neoadjuvant chemotherapy plus radiotherapy with radiotherapy alone in patients with locoregionally advanced nasopharyngeal carcinoma. J Clin Oncol. 2001; 19:1350-1357.

22. Tan T, Lim WT, Fong KW, Cheah SL, Soong YL, Ang MK, Ng QS, Tan D, Ong WS, Tan SH, Yip C, Quah D, Soo KC, et al. Concurrent chemo-radiation with or without induction gemcitabine, Carboplatin, and Paclitaxel: a randomized, phase $2 / 3$ trial in locally advanced nasopharyngeal carcinoma. Int J Radiat Oncol Biol Phys. 2015; 91:952-960.

23. Sun Y, Li WF, Chen NY, Zhang N, Hu GQ, Xie FY, Sun Y, Chen XZ, Li JG, Zhu XD, Hu CS, Xu XY, Chen YY, et al. Induction chemotherapy plus concurrent chemoradiotherapy versus concurrent chemoradiotherapy alone in locoregionally advanced nasopharyngeal carcinoma: a phase 3, multicentre, randomised controlled trial. Lancet Oncol. 2016; 17:1509-1520.

24. Chua DT, Ma J, Sham JS, Mai HQ, Choy DT, Hong MH, Lu TX, Min HQ. Long-term survival after cisplatinbased induction chemotherapy and radiotherapy for nasopharyngeal carcinoma: a pooled data analysis of two phase III trials. J Clin Oncol. 2005; 23:1118-1124.

25. Chen YP, Mao YP, Zhang WN, Chen L, Tang LL, Li WF, Liu X, Zhou GQ, Guo R, Sun Y, Kang TB, Zeng MS, Ma J. Prognostic value of wait time in nasopharyngeal carcinoma treated with intensity modulated radiotherapy: a propensity matched analysis. Oncotarget. 2016; 7:14973-14982. https://doi.org/10.18632/oncotarget.7789.

26. Ribassin-Majed L, Marguet S, Lee AW, Ng WT, Ma J, Chan AT, Huang PY, Zhu G, Chua DT, Chen Y, Mai HQ,
Kwong DL, Cheah SL, et al. What Is the Best Treatment of Locally Advanced Nasopharyngeal Carcinoma? An Individual Patient Data Network Meta-Analysis. J Clin Oncol. 2016; 35:498-505.

27. Chen Y, Sun Y, Liang SB, Zong JF, Li WF, Chen M, Chen L, Mao YP, Tang LL, Guo Y, Lin AH, Liu MZ, Ma J. Progress report of a randomized trial comparing long-term survival and late toxicity of concurrent chemoradiotherapy with adjuvant chemotherapy versus radiotherapy alone in patients with stage III to IVB nasopharyngeal carcinoma from endemic regions of China. Cancer. 2013; 119:2230-2238.

28. Chen L, Hu CS, Chen XZ, Hu GQ, Cheng ZB, Sun Y, Li WX, Chen YY, Xie FY, Liang SB, Chen Y, Xu TT, Li B, et al. Concurrent chemoradiotherapy plus adjuvant chemotherapy versus concurrent chemoradiotherapy alone in patients with locoregionally advanced nasopharyngeal carcinoma: a phase 3 multicentre randomised controlled trial. Lancet Oncol. 2012; 13:163-171.

29. International Nasopharynx Cancer Study Group, VUMCA I Trial. Preliminary results of a randomized trial comparing neoadjuvant chemotherapy (cisplatin, epirubicin, bleomycin) plus radiotherapy vs. radiotherapy alone in stage $\operatorname{IV}(>$ or $=\mathrm{N} 2, \mathrm{M} 0)$ undifferentiated nasopharyngeal carcinoma: a positive effect on progression-free survival. Int J Radiat Oncol Biol Phys. 1996; 35:463-469.

30. Liang H, Xiang YQ, Lv X, Xie CQ, Cao SM, Wang L, Qian CN, Yang J, Ye YF, Gan F, Ke LR, Yu YH, Liu GY, et al. Survival impact of waiting time for radical radiotherapy in nasopharyngeal carcinoma: A large institution-based cohort study from an endemic area. Eur J Cancer. 2017; 73:48-60.

31. Chen Y, Liu MZ, Liang SB, Zong JF, Mao YP, Tang LL, Guo Y, Lin AH, Zeng XF, Ma J. Preliminary results of a prospective randomized trial comparing concurrent chemoradiotherapy plus adjuvant chemotherapy with radiotherapy alone in patients with locoregionally advanced nasopharyngeal carcinoma in endemic regions of china. Int J Radiat Oncol Biol Phys. 2008; 71:1356-1364.

32. Austin PC. The relative ability of different propensity score methods to balance measured covariates between treated and untreated subjects in observational studies. Med Decis Making. 2009; 29:661-677.

33. Peng H, Guo R, Chen L, Zhang Y, Li WF, Mao YP, Sun Y, Zhang F, Liu LZ, Lin AH, Ma J. Prognostic Impact of Plasma Epstein-Barr Virus DNA in Patients with Nasopharyngeal Carcinoma Treated using IntensityModulated Radiation Therapy. Sci Rep. 2016; 6:22000.

34. Lin JC, Wang WY, Liang WM, Chou HY, Jan JS, Jiang RS, Wang JY, Twu CW, Liang KL, Chao J, Shen WC. Longterm prognostic effects of plasma epstein-barr virus DNA by minor groove binder-probe real-time quantitative PCR on nasopharyngeal carcinoma patients receiving concurrent chemoradiotherapy. Int J Radiat Oncol Biol Phys. 2007; 68:1342-1348.

35. Chan AT, Lo YM, Zee B, Chan LY, Ma BB, Leung SF, Mo F, Lai M, Ho S, Huang DP, Johnson PJ. Plasma Epstein- 
Barr virus DNA and residual disease after radiotherapy for undifferentiated nasopharyngeal carcinoma. J Natl Cancer Inst. 2002; 94:1614-1619.

36. Pan JJ, Ng WT, Zong JF, Chan LL, O'Sullivan B, Lin SJ, Sze HC, Chen YB, Choi HC, Guo QJ, Kan WK, Xiao YP, Wei X, et al. Proposal for the 8th edition of the AJCC/ UICC staging system for nasopharyngeal cancer in the era of intensity-modulated radiotherapy. Cancer. 2016; 122:546-558.
37. Xu JH, Guo WJ, Bian XH, Wu JF, Jiang XS, Guo YS, He X. A comparative study of locoregionally advanced nasopharyngeal carcinoma treated with intensity modulated irradiation and platinum-based chemotherapy. Cancer Radiother. 2013; 17:297-303. 